\title{
Difficulté, intérêt, nécessité d'une démarche comparative dans la perspective du développement durable
}

\author{
VÉRONIQUE DE LA BROSSE
}

S'il est difficile de nier l'intérêt intellectuel d'une Sdémarche comparative quel qu'en soit l'objet, dans une optique scientifique le comparatisme ne se conçoit qu'à la condition d'être fondé sur une solide argumentation préalable. En géographie en particulier cela s'explique aussi bien par les traditions monographiques de la discipline que par ses orientations systémiques ou fonctionnalistes plus récentes. Plus généralement, le comparatisme dérange les sciences sociales - et les sociètés elles-mêmes - dans la mesure où il met en présence des notions d'identité et d'altérité qui, trop souvent considérées comme allant de soi, fondent le discours social aussi bien que les méthodes scientifiques.

Le comparatisme perturbe cette conception de l'Autre, qui remonte au moins au $x v \|^{\mathrm{e}}$ siècle, et sur laquelle est solidement fondée depuis la Troisième République toute entreprise de redéfinition de l'identité d'autrui, qu'elle ait pour nom colonisation, développement, aménagement du territoire. L'Autre, c'est celui qui est privé des Lumières. C'est tout d'abord le rustre de Voltaire, ce paysan français inconnu, vêtu de peaux de bête, inférieur au bon sauvage dans la hiérarchie la classification - des êtres (J. de Viguerie, 1993). Les êtres une fois classifiés, justement, ne peuvent plus être comparés. La différence proclamée, intériorisée, devient une cloison mentale qui permet de se croiser sans se voir. L'Autre n'est certes pas votre prochain, et vous pouvez admettre en toute inconscience qu'il ait à subir tout ce que vous ne voudriez pas qu'on vous fit.

Les critères de la classification sont une de ces zones d'ombre des sciences sociales oủ la démarche scientifique est le plus facilement entâchée d'arbitraire et susceptible de refléter les lieux communs du discours vulgaire. L'ordonnancement des sociétés humaines est par excellence une démarche politique, ne pas la reconnaitre comme telle c'est laisser le champ libre à l'idéologie qui légitime l'ordre social par l'ordre naturel. La notion de classification, empruntée aux sciences biologiques, suppose des espèces différentes par nature, et c'est cela qui fonde implicitement la démarche a priori absurde consistant à classer avant d'avoir comparé. L'héritage de Lucien Lévy Bruhl pèse encore sur la démarche anthropologique. La quête de l'universel à travers le filtre des cultures particulières, la possibilité de concevoir l'échange inter-culturel, la notion même de culture en définitive restent fortement diminuées par les réminiscences de la mentalité primitive, " racisme métaphysique - $(R$. Lourau, 1992) qui justifie aussi bien l'exploitation néocoloniale que l'enfermement dans la différence - dans l'indifférence.

La notion d'équité, mise en avant dans la problématique du développement durable, interpelle cette tradi- tion scientifique de la classification et du cloisonnement. L'équité versus l'égalité, débat juridique classique, se situait jusqu'ici au sein d'une même culture ou du moins dans un contexte où les sujets mis en présence sont censés avoir des références communes. Comment apprécier l'équité entre des sujets relevant de contextes a priori différents et qui se définissent par défaut, c'est-à-dire : il, elle, est l'Autre, ce que je ne suis pas, ce que je ne sais pas?

Ceci s'applique à l'équité intergénérationnelle aussi bien qu'intragénérationnelle. Dans les deux cas intervient la dimension du changement technologique, du niveau de développement, qui transforme au point de rendre méconnaissable, au point de mettre en péril la notion de patrimoine et avec elle celle de continuité, de communauté, de reproduction.

La question posée à propos de l'équité intergénérationnelle - comment puis-je savoir ce dont les générations futures auront besoin et ce qu'elles tiendront pour précieux ? - sous-entend que le progrès technique toujours plus rapide transforme les milieux et les mentalités de manière si radicale que la génération suivante pourrait être d'une autre espèce, en quelque sorte, dépourvue de toute communauté de vue avec la génération présente. De même, l'avancée inégale du progrès technique au sein d'une même génération la sépare en deux espèces, les développés et les autres.

Quelles sont, en géographie, les critères permettant d'écarter ou au contraire de légitimer a priori une option comparative ? La réponse à cette question met en jeu l'identité territoriale telle qu'elle est appréhendée, dans chaque cas particulier et sur un plan théorique, par le géographe.

Les découpages territoriaux - idéels ou réels conçus dans une perspective politique et macroéconomique ont cru pouvoir assigner aux territoires ainsi remodelés, en même temps qu'une fonction nouvelle, une identité à venir. La notion de patrimoine est venue alors fonder le besoin de permanence, de reproduction de soi pour soi, des sociétés locales. De même la notion de pays, chère à la géographie classique, resurgit comme porteuse d'une identite territoriale définie localement. Bien entendu, ces notions sont rapidement prises en main et remaniées, comme les territoires eux-mêmes, par une autorité extérieure région, État, institution internationale. Une réflexion comparative apporterait un éclairage utile sur bien des points mis en question dans ce jeu à rebondissements entre le local et le global. La démarche comparative qui nous paraît le plus nécessaire est celle qui met en perspective des terrains situés en Europe d'une part, dans les anciennes colonies ou zones d'influence européennes d'autre part.
Veronique de La Brosse coordonne un programme de recherche sur les grands aménagements hydroagricoles en Afrique de l'Est et en Europe méditerranéenne et orientale soutenu par le CNRS (programme PIR-EVS).

Cette expérience nourrit une réflexion personnelle sur la problématique du développement durable 
En effet, l'intérêt de la démarche comparative réside dans son caractère perturbateur et controversé. C'est seulement dans la mesure où il est prêt à remettre en cause au besoin les postulats sur lesquels repose son appréhension de son terrain d'origine que le chercheur en tirera parti. II lui faudra en quelque sorte une lucidité équivalente à celle des africanistes qui, dans l'ouvrage collectif dirigé par J.-L. Amselle et E. M'Bokolo (Au cœur de l'ethnie, 1985), ont été amenés à démontrer le caractère artificieux de la notion d'ethnie. Peut-être l'identité territoriale telle qu'elle est aujourd'hui conçue et étudiée sortira-t-elle décomposée de la même façon au terme d'une démarche qui privilégie, dans la recherche de l'identité, le rapport à l'autre.

Dans une perspective historique et à l'échelle spatiale et temporelle de l'empire romain ou du monde méditerranéen arabisé, la problématique qui consiste à vouloir comparer l'adaptation locale de modèles techniques centralisés et centralisateurs apparaît pertinente, en particulier à cause du processus d'indigénisation auquel se sont prêtées de manière caractéristique ces deux cultures. Le problème se pose lorsqu'il s'agit de mettre en parallèle des sociétés industrielles et préindustrielles, ou plus exactement des sociétés locales incluses dans un état industrialisé, et d'autres faisant partie d'un pays dont l'économie reste essentiellement rurale et informelle. La notion de société locale peut-elle prétendre recouvrir, même partiellement, des réalités comparables dans les pays du * Sud * et dans les pays du " Nord " ?

Cependant le pouvoir d'intervention technique dont dispose l'État moderne apparaît sans commune mesure avec ce qu'il a jamais pu être dans le passé. Les ressources et la capacité d'adaptation des cultures techniques locales apparaissent désormais dérisoires devant les transformations imposées par la puissance étatique.

Face à un grand aménagement hydroagricole, la société locale se trouve marginalisée sur le plan territorial et culturel aussi bien que sur le plan démographique. Une constante de ces modèles de développement, qu'ils s'implantent sur un territoire métropolitain, colonial ou périphérique, est leur recours massif à une main d'œuvre extérieure par l'intermédiaire de laquelle sintroduisent les nouvelles techniques et un nouveau système agrofoncier. Dans nombre de cas, d'importants déplacements de population précèdent la mise en eau de l'ouvrage.

Que reste-t-il donc à comparer, si ce n'est le rapport des ingénieurs avec le milieu physique, l'adaptation du modèle technique à différents environnements ?

Mais ici encore, on se trouve en présence d'une formidable entreprise d'uniformisation du milieu : toute identité locale forte pose problème, et il est désormais possible de circonvenir d'emblée et très rapidement les éléments non contrôlables, qu'il s'agisse du milieu physique ou humain.

L'intérêt d'une approche comparative du rapport entre le local et le global tel qu'il s'exerce dans le cadre de la grande hydraulique agricole apparait donc limité par deux types de considérations. Tout d'abord, la nature radicalement différente des deux composantes de ce rapport - l'État et les sociétés locales - et donc du rapport lui-même, dans un contexte ouest-européen et africain, d'autre part le déséquilibre introduit par le progrès technologique au service de l'État, lequel a pour conséquence d'annihiler pratiquement les sociétés locales comme interlocuteurs ou partenaires des autorités chargées d'aménager leur territoire.

On s'aperçoit que ces deux objections s'annulent mutuellement. En effet, si l'on tient pour acquis le fait que dans le contexte occidental les sociétés locales conservent un pouvoir de négociation face à la puissance étatique - ce qui est loin d'être certain, au moins lorsque l'enjeu pour l'État est suffisamment important -, il faut démontrer d'une part que les identités territoriales sont aussi marquées sinon plus dans les pays industrialisés que dans les pays * en développement . à moins de s'en tenir à une conception purement institutionnelle du rapport entre l'État et la région. Cette idée, intéressante en soi, suppose une démarche comparative. Elle remet en cause la notion de sociétés traditionnelles couramment utilisée pour qualifier les sociétés locales des pays non-occidentaux, qui sousentend une identité territoriale forte, un enracinement dans l'espace, une entité échappant au moins partiellement à l'État-nation.

Si par ailleurs on considère que le modèle technocratique est suffisamment puissant pour niveler les particularismes locaux, idée implicitement véhiculée par ce modèle mais rarement exprimée, il faut chercher la cause de ses dysfonctionnements ailleurs que dans la résistance des sociétés locales. Indépendamment de son interaction avec un milieu extérieur, le modèle estil viable ou n'est-il pas condamné à l'échec par sa propre dynamique interne?

Il faut aborder ici, à côté de la pertinence du savoir technique lui-même, la question de la cohabitation des cultures techniques et des cultures tout court à l'intérieur même des institutions chargées de mettre en œuvre les politiques de développement et d'aménagement du territoire.

II s'avère que la coupure entre le local et le global est purement intellectuelle. Dans la réalité ces deux ordres de références s'interpénètrent plus qu'ils ne se superposent. La nébuleuse technocratique est composée de corps de métiers dont les pratiques et les représentations relèvent tout autant de leurs - traditions * respectives que d'une quelconque logique technique et l'idéal technocratique recouvre en fait une multiplicité d'approches et de positionnements. Leurs traditions respectives ne sont pas sans rapport avec le recrutement social de ces institutions : des ingénieurs d'origine paysanne, par exemple, n'auront pas la même approche du développement rural que des cadres nés en ville - ni, peut-être, la même position institutionnelle -, et ce clivage ne recoupe pas forcément celui entre pays du Sud et pays industrialisés.

Les dissensions entre institutions chargées de mettre en œuvre le modèle technique peuvent faire imploser ce modèle, et ces dissensions relèvent d'un positionnement différent de ces institutions vis-à-vis de l'État et des sociétés locales. On identifie là une variable susceptible de nous éclairer sur la nature de l'État et sur l'identité locale - notamment la capacité de cette dernière de s'infiltrer dans l'idéologie technocratique. Apparente au point de prêter à la caricature dans les 
pays en développement - sous la forme bien connue de la solidarité clanique, cette capacité pourrait être également mise en lumière dans nos sociétẻs unitaires. La machine technocratique ne serait-elle pas le lieu privilégié oủ s'affrontent les particularismes et les corporatismes de toutes sortes, ceux-là même que cet appareil s'est attaché à niveler au sein de la société ?

Pour en revenir aux sociétés locales dans la perspective du développement durable, le constat préalable à toute problématique nous paraît devoir être extrêmement pessimiste. Le discours sur la capacité d'adaptation des cultures et des savoirs techniques locaux, et même celui sur la prise en compte des spécificités locales et sur l'enracinement de toute action de développement dans un milieu particulier, se heurte inéluctablement à la question de savoir s'il existe encore des sociétés locales, et si la conception moderne du pouvoir et les moyens modernes d'exercice du pouvoir - idéologiques, institutionnels, techniques - laissent une possibilité quelconque à l'existence d'un rapport du global au local qui ne soit pas de domination absolue.

La grande entreprise économique qui consiste à déstructurer les sociétés traditionnelles pour, d'une part, en " extraire le facteur travail - (K. Polanyi, 1944) c'est-à-dire les transformer en réservoirs de main d'œuvre et, d'autre part, en conquérir le marché, seraitelle en certains points du globe restée inachevée ? Ou bien est-il possible, quoiqu'il en soit, de revenir en arrière, de restituer à ces sociétés dépossédées leur identité perdue?

Est-il possible de croire à une action de l'État moderne qui consisterait à protéger et à défendre ces identités locales contre la - tendance lourde * de la mondialisation dont il a été jusqu'ici l'un des promoteurs ? Mais l'État lui-même ne serait plus, selon certains, qu'un résidu de ces identités périmées qu'il a contribué à détruire.

Nous sommes entrés dans l'ère des institutions internationales véhicule d'une pensée globale, pensée intégratrice, pensée hybride oủ tous les discours, en définitive, tiennent leur place, du tiers-mondisme au technocratisme en passant par l'écologisme. La problématique du développement durable en est lillustration la plus intéressante, et comme telle elle fournit un cadre propice, mieux encore elle appelle, au comparatisme.

En effet la grille de concepts qui structure la réflexion sur le développement durable, issue des travaux d'une commission des Nations unies - laquelle a surtout, diton, effectué un travail de synthèse - est applicable aussi bien aux pays dits en développement qu'aux pays industrialisés. Les notions d'équité spatiale et temporelle, de gestion patrimoniale des ressources naturelles se diffusent actuellement dans le monde entier par les canaux institutionnels secondaires et tertiaires que sont les agences spécialisées des Nations unies, les gouvernements et leurs agences, les organisations non-gouvernementales. Dans le cas français, ces notions se retrouvent, après avoir transité à l'échelon européen, dans les dernières lois d'aménagement du territoire (F. Lefèbvre 1995), dans les directives récentes de la Datar ${ }^{1}$, dans tout un travail d'exégèse réalisés par les collaborateurs scientifiques des différentes instances de l'État.

Il sera intéressant d'observer comment la notion d'équité spatiale pourra se concilier avec la spécialisation régionale dont il est prévu qu'elle aille en s'accroissant au sein de I'Union européenne. Des instruments de comparaison suffisamment sophistiqués devront être élaborés pour définir les critères d'un développement tout à la fois disparate et équitable.

La même démarche est nécessaire à l'échelle du monde, dans la mesure oủ le développement durable réclame un rééquilibrage des rapports Nord-Sud comme condition d'une approche patrimoniale des ressources de la planète. Léquité entre régions du Nord et du Sud suppose un mode de développement qui ne se limite pas aux transferts de capitaux ou de techniques sous forme d'aide plus ou moins conditionnelle entre un centre * central - et un centre - périphérique *, elle demande à ce que les - acteurs locaux du développement - soient d'emblée pris en considération et impliqués dans le processus de " coopération ". Ici se pose la question de la démocratie locale, c'est-à-dire de la démocratie tout court.

La démocratie locale étant la grande question en suspens dans les sociétés développées qui servent de modèle (le local, c'est-à-dire le réel, continue, plus que jamais, à faire problème), de quelles méthodes disposent les planificateurs internationaux pour appréhender le local dans les - pvd - ? On revient ici à la question des moyens techniques de l'exercice moderne du pouvoir. Pour pouvoir envisager un autre type de rapport au local, il faudrait d'abord disposer de techniques avec lesquelles les sociétés locales puissent non seulement cohabiter, ce qui est déjà difficile, mais interagir. Ici intervient la question des * technologies douces ", déjà mise en avant dans le discours sur l'écodéveloppement des années 1970 , et qui se pose aujourd'hui de manière plus générale et plus pressante.

\section{Bibliographie}

Amselle J-L, M Bokolo E.S. (dir.) (1985). Au cœeur de l'ethnie : ethnies, tribalisme et Etat en Afrique. La Découverte, Paris

Lefébvre F. (1995). - Les pays selon la loi dorientation pour l'aménagement et le développement du territoire - Rev. Fr. de droit administratif 11, 5 , 884-892

Lourau R. (1992). - Lucien Lévy-Bruhl : Un acte manqué Nord-Sud ·. LHomme et la societe 105-106, 155-168

de Viguerie J. (1993). - Des Lumières et des peuples * Revue historique 587, 1 Délégation à 1993, 161-189.

l'aménagement du

Polanyi K. (1983). La Grande Transformation aux origines politiques et econo- territoire et à l'action miques de notre temps. Gallimard, Paris régionale. 